\title{
Fruit Freshness Detection Using Raspberry PI
}

\author{
Krithika Jayasankar $^{1}$, Karthika $\mathrm{B}^{2}$, Jeyashree $\mathrm{T}^{3}$, Deepalakshmi $\mathrm{R}^{4}$, Karthika $\mathrm{G}^{5}$ \\ ${ }^{1,2,3,4}$ UG student, Department of Electronics and Instrumentation Engineering, RMD Engineering \\ College, Kavaraipettai, Tamilnadu, India \\ 5, Assistant professor, Department of Electronics and Instrumentation Engineering, \\ RMD Engineering College, Kavaraipettai,Tamilnadu, India
}

\section{ABSTRACT:}

This paper sheds light on the advancements made in the agricultural industry. Digital image processing techniques are now widely used for the maturity estimation of fruits. This work aimed to study and analyze the various algorithms and feature extraction techniques that are now used for the extracting features from the captured digital images. Thus, it is important for the suppliers to label the quality of fruits. In this paper, we are inspecting the quality of fruits based on size, shape and color and also by its weight. All these algorithms are implemented using RASPBERRY PI development board which will become an independent and cost effective system. All the interfacing of the components will be carried out and will make a cost effective embedded system prototype for the determination of size, shape and color of the fruit. Same system can be utilized for other fruits also. Advantages and disadvantages of various classifiers have been classified. It was observed that for achieving high accuracy a compromise had to be made with high computational complexity.

KEYWORDS: Raspberry Pi, Digital Image Processing, Embedded.

\section{INTRODUCTION}

This paper describes how the freshness of fruit can be determined using Raspberry $\mathrm{Pi}$.As we know food plays a vital role in human survival. Due to improper handling and storage might cause food poisoning. This happens because most of the bacteria are invisible and undetectable. Moreover, these bacteria typically don't change the taste, smell or the outlook of fruit. Therefore, we should analyze the methods to prevent food poisoning and help to consume fresh edibles. This project has a detector system which detects the freshness of fruits using raspberry pi. Whenever the fruit is being picked, it should be placed in a conveyor belt, where it is passed through the sensor unit which can detect and display the complete freshness status of the fruit. The recent application and development of image analysis in quality evaluation of products in the field of agricultural and food. Images are the important sources of data and information in the agricultural science. The basic concepts and technologies associated with computer vision system and automatic vision based technology, tool used in image analysis and automated sorting and grading is highlighted. The proposed system starts 
the process by capturing the fruit image by using Raspberry $\mathrm{Pi}$. Then, the image is transmitted to the processing level where the fruit's characteristics like color, shape and size of fruit samples are extracted. After that by artificial neural network fruit images are going through the training and testing section. In this proposed paper neural network is used to detect the shape, size and color of fruit and with the combination of these three features the results obtained are very promising. The quality of different fruits is detected and the percentage of fruits is displayed in the monitor.

\section{EXISTING SYSTEM}

A smart sensor plate is utilized, where the sensor plate is utilized to recognize the freshness of family unit things like meat, cooked things. Brilliant plate is a level plate comprising of an assortment of sensors that are enacted relying upon the sustenance thing. This plate is put in the utensil and board can be utilized to choose the sort of sustenance thing. Facilitate the functionalities is executed into gadget through advanced mobile phones. A portable application is created to get information from PDAs to produce progress ahead of time in regards to the status of the sustenance thing. The nourishment thing to be recognized is put in an utensil and the savvy plate is put inside the vessel subsequent to making the required setting. The sensors in the sensor plate are enacted relying upon the nourishment things and afterward decide the nature of sustenance things. The client input is java based android application that can be utilized to turn on the shrewd plate and indicate the nourishment thing. The application is utilized to furnish availability with advanced mobile phones by means of blue tooth and select the nourishment thing whose quality to be checked. In view of sustenance thing sensor plate enacts the specific sensor for testing the microcontroller gets the client input and furthermore gets the contribution from sensor plate and decides the freshness level of nourishment thing in light of predefined calculation. The yield of LED decides if the sustenance is great or awful. Green Led demonstrate the sustenance is great or the other way around. The freshness level of the sustenance thing is shown utilizing easy to use show.

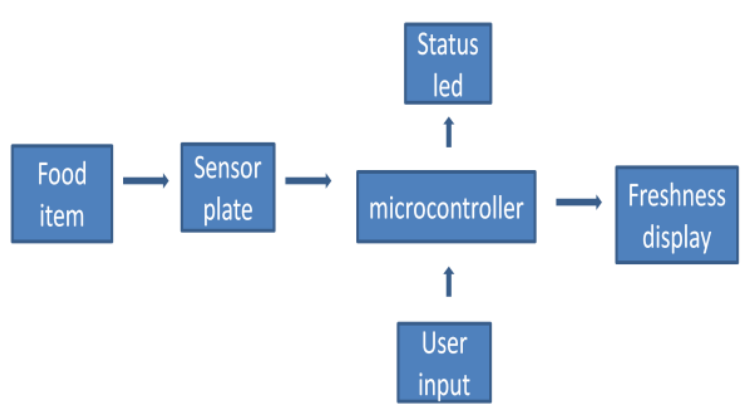

\section{PROPOSED SYSTEM}

The main intension of this paper is to introduce a new prototype for the detection of freshness in fruits. In current scenario, people don't analyze how fresh the fruits and vegetables they are consuming. The prototype comprises of a conveyor belt setup 
which includes various sensors like proximity sensor, load cell and gas sensor.

\section{COMPONENTS}

The sequence of components and concepts required in this paper are listed below.
a) Raspberry pi $0 \mathrm{~W}$
b) Digital Image Processing
c) Proximity sensor
d) Load cell
e) Gas sensor

\section{a) RASPBERRY PI OW}

Raspberry Pi is a progression of little single-board PCs created in the United Kingdom by the Raspberry Pi establishment to advance the educating of essential software engineering in schools and in creating nations. Among different arrangement of Raspberry PI this task has utilized Raspberry Pi 0 W. This model is an innovative, smaller than usual variant of the Raspberry Pi that therapists the board down to about a size of a stick of gum, however one such issue with it is the absence of remote highlights. The full particulars of Raspberry Pi $0 \mathrm{~W}$ are $1 \mathrm{GHz}$, Single core CPU

$\begin{array}{ll}\checkmark & 512 \text { MB RAM } \\ \checkmark & \text { Mini HDMI and USB On-The- } \\ & \text { Go ports } \\ \checkmark & \text { Micro USB power } \\ \checkmark & \text { HAT-compatible 40-pin header } \\ \checkmark & \text { Composite video and reset } \\ & \text { headers } \\ \checkmark & \text { CSI camera connector } \\ \checkmark & \text { 802.11n wireless LAN } \\ \checkmark & \text { Bluetooth 4.0 }\end{array}$

Vol 1 (10), April 2018, www.ijirase.com

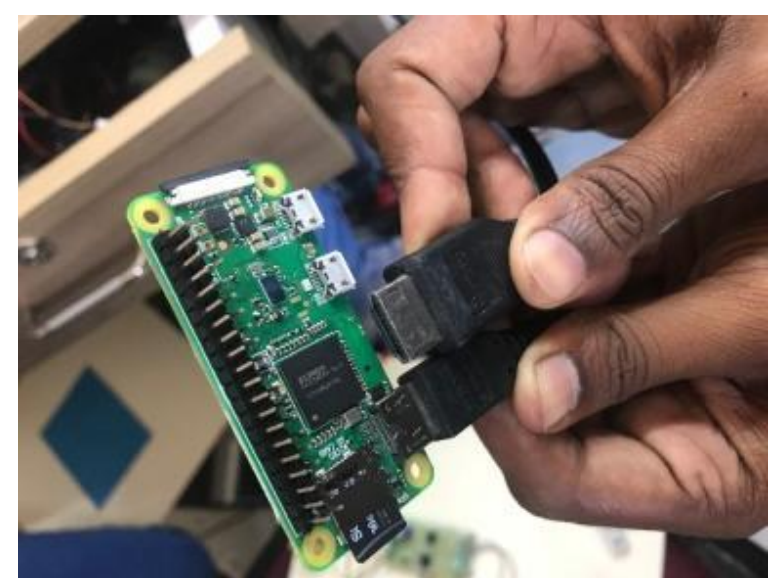

The required working voltage is $3.3 \mathrm{~V}$. The working frequency of the processor is $800 \mathrm{MHz}$.

\section{b) DIGITAL IMAGE PROCESSING}

Modernized Image Processing thought expect a fundamental part in this paper. Propelled Image Processing is the use of PC estimations to perform picture getting ready on modernized pictures. As a subcategory or field of modernized banner getting ready, propelled picture taking care of has various central focuses over basic picture planning. Taking care of is done by using open CV technique. Advanced Image Processing oversees control of modernized pictures through a propelled PC. It is a subfield of signs and systems yet base particularly on pictures. Dive revolves around working up a PC structure that can perform getting ready on a photo. The commitment of that structure is a propelled picture and the system method that photo using viable computations, and gives a photo as a yield. The computation performed here is the edge distinguishing proof count. One such for the most part used application for getting ready propelled pictures. 


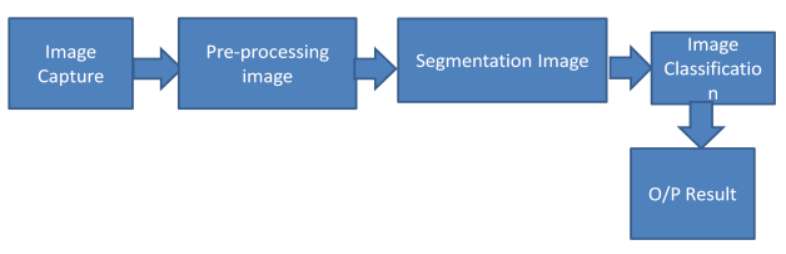

\section{c) PROXIMITY SENSOR}

IR sensor work by using a specific light sensor to distinguish a select light wavelength in the Infra-Red (IR) go. By using a LED which conveys light at a vague wavelength from what the sensor is hunting down, you can look at the power of the got light. Exactly when the challenge is close to the sensor, the light from the LED ricochets off the inquiry and into the light sensor. This results in tremendous jump in the power, which we unquestionably know can be perceived using a breaking point.

\section{d) LOAD CELL}

At the point when steel chamber is subjected to a power, it tends to change in measurement. On this barrel, if the strain measures are reinforced, the strain check additionally is extended or compacted, causing an adjustment in its length and distance across. This adjustment in measurement of the strain check makes its protection change. This adjustment in Resistance or yield voltage of the strain check turns into a measure of connected power.

\section{e) GAS SENSOR}

A gas marker is a device that recognizes the closeness of gases in a zone, consistently as a noteworthy part of a prosperity structure.
This sort of equipment is used to recognize a gas spill or diverse surges and would interface have the capacity to with a control structure so a system can be thusly shut down. A gas locator can sound an alert to managers in the zone where the break is occurring, allowing them to take off. This sort of device is basic in light of the way that there are various gases that can be pernicious to regular life, for instance, individuals or animals. If any gas is been sprinkled in any such natural item then this sensor helps in recognizing it by giving an alert or indicating it in the screen.

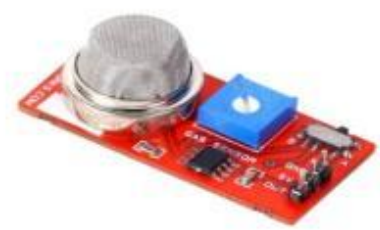

\section{IMPLEMENTATION PROCESS}

The program is dumped into the Raspberry Pi OW board. Caspian OS is the OS involved. The proposed system starts the process by capturing the fruit or vegetable image. Then, the image is transmitted to the processing level in open $\mathrm{CV}$ where the fruit features like color, shape and size of fruit samples are extracted. In this proposed paper open cvmethod is used to detect shape, size and color of fruit and with the combination of these three features the results obtained are very promising. Captured image is segmented using edge detection algorithms in order to find the defected fruit. Load cell is used to identify the weight of the placed samples and it takes an average of the weight and this is one of the parameter taken under consideration for the identification of the freshness of fruit. Gas sensor is used to identify the gas present or sprayed in the fruits. Freshness 
ISSN(Online) : 2456-8910

International Journal of Innovative Research in Applied Sciences and Engineering (IJIRASE) Volume 1, Issue 10, DOI: 10.29027/IJIRASE.v1.i10.2018.202-208, April 2018

percentage for different fruits are also identified and displayed through LCD display.

BLOCK DIAGRAM

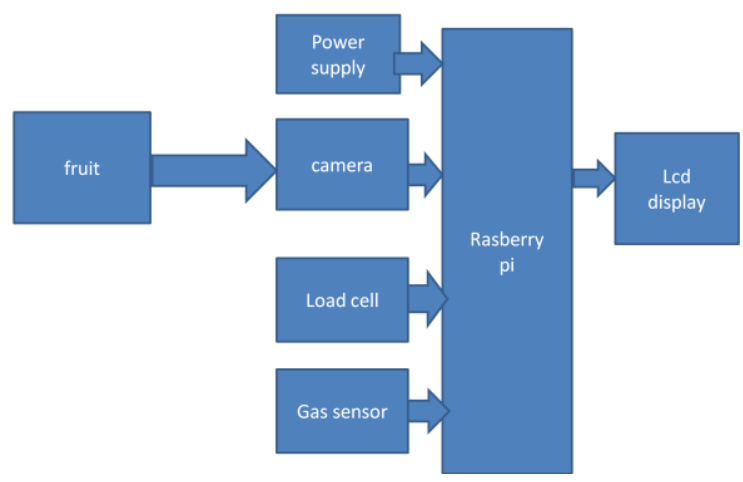

\section{RESULTS}
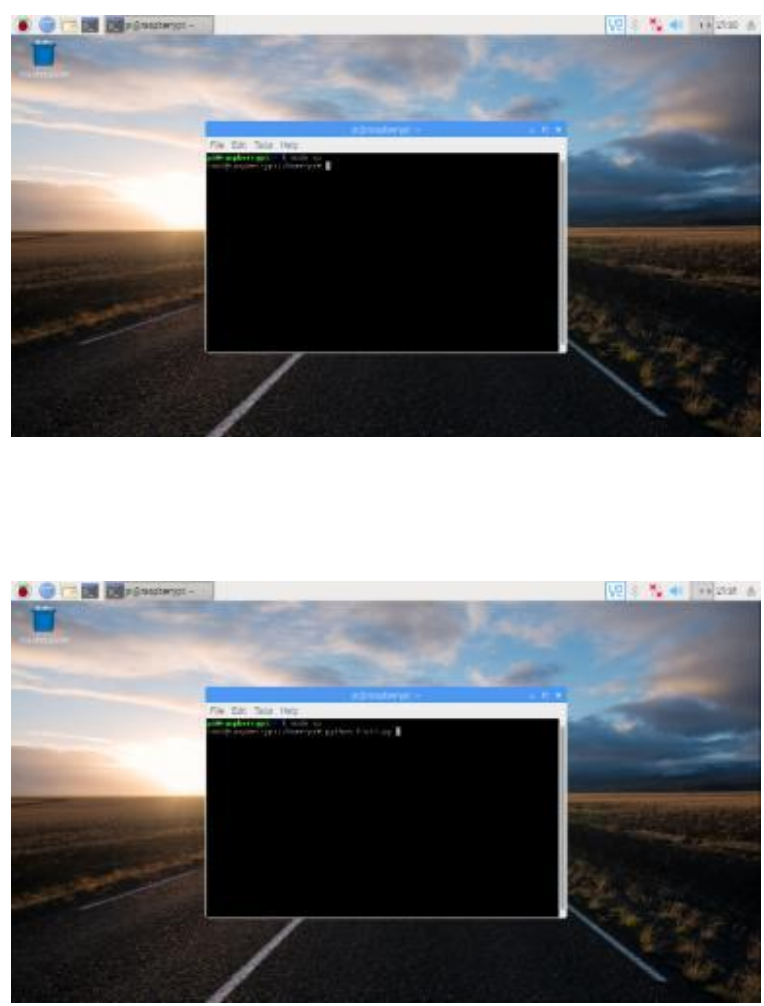
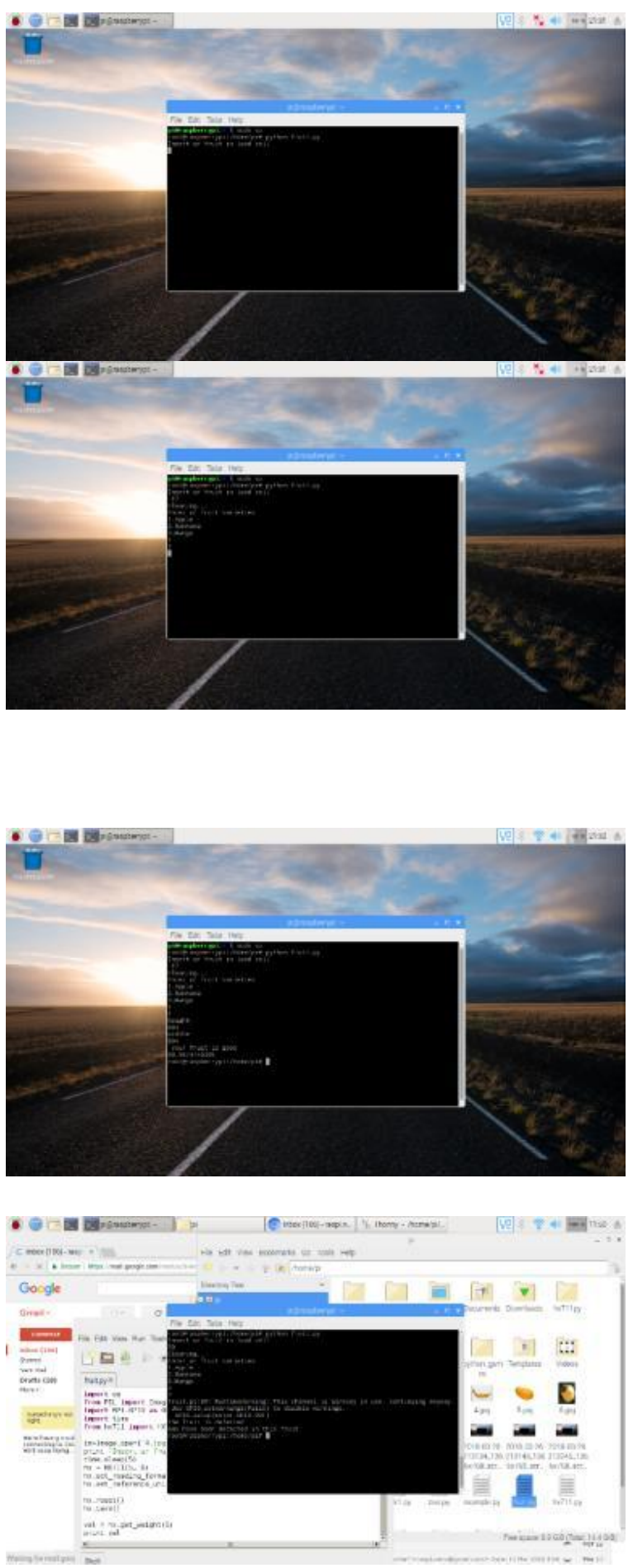


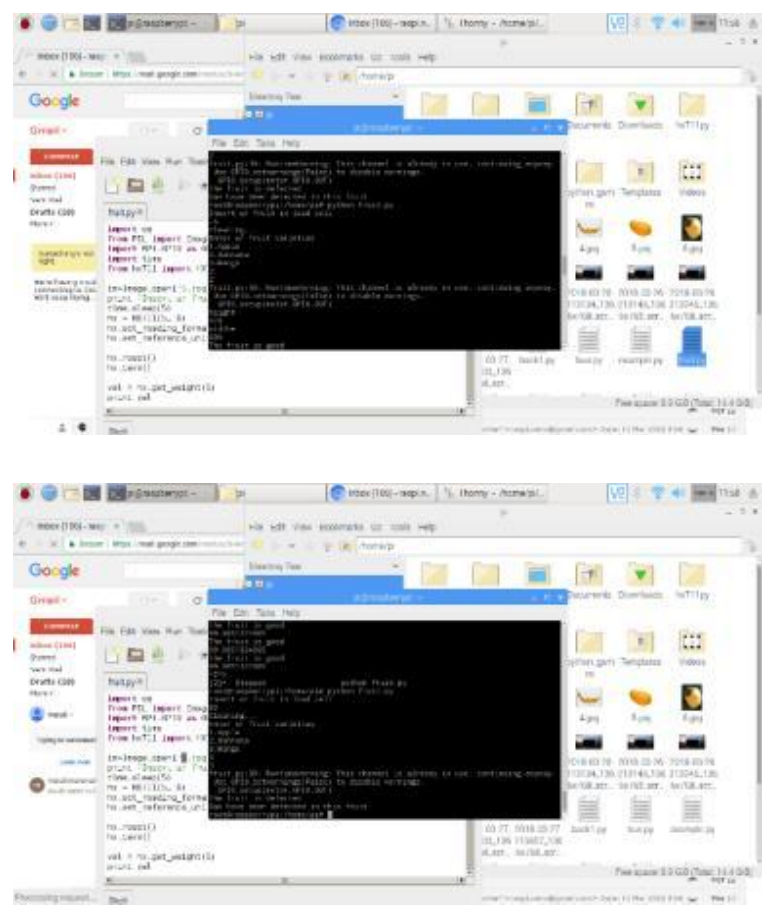

The result displayed here is the performance of Digital Image Processing. So considering the height and width (i.e) the two main parameters a limited percentage of freshness is updated. Then the load is given and after the weighing process again it updates the percentage of freshness of fruit. So these are the various characteristics considered for the detection of the quality of fruits.

\section{PROTOTYPE MODEL}

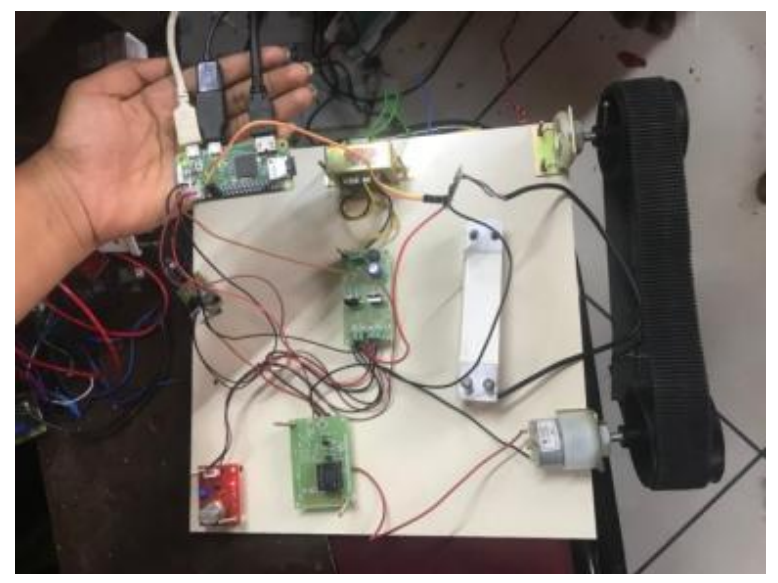

Vol 1 (10), April 2018, www.ijirase.com

\section{ADVANTAGES}

- Pollution due to wastes can be reduced.

- Any person consuming the edible is aware of the quality of fruits or vegetables before the usage.

- It can easily check the freshness of any edible.

\section{DISADVANTAGES}

- Since this idea is based upon image processing there is a drawback in detecting the exact freshness status of the images with lower resolution.

- Time taken for the conveyor belt to move fruits is more.

- Internal scanning of edible by means of rays will result in damaging.

\section{CONCLUSION}

This method will be efficient enough because before the intake of an edible this process automatically displays the entire status of any fruits and vegetables. The consumer can choose any quality of an edible depending upon their own needs. So by obtaining this process the food wastage can be reduced and will be able to achieve pollution less environment.

\section{FUTURE ADVANCEMENTS}

As discussed above the method of detecting the freshness of edible deals in image processing reveals the quality only by outer appearance. Hence this technique is 
successful about $80 \%$ in freshness detection.

The plan works on still better by advancing the process in viewing the internal structure contactless. It can be achieved by cargo scanning methodology. With this designing displaying the originality of the product is even more accurate.

\section{REFERENCES}

1) H. Ayasso and A. MohammadDjafari, "Joint NDT Image Restoration and Segmentation Using GaussMarkov-Potts Prior Models and Variational Bayesian Computation," IEEE Transactions on Image Processing, vol. 19, no. 9, pp. 2265-77, 2010. [Online]. Available: IEEE Xplore, http://www.ieee.org. [Accessed Sept. 10, 2010].

2) ImanMorsi, A Microcontroller Based on Multi Sensors Data Fusion and Artificial Intelligent Technique for Gas Identification, The 33rd IEEE Industrial Electronic Society(IECON), Taiwan, Nov.5, 2007.

3) ImanMorsi, Discrimination of Some Atmospheric Gases Using an Integrated Sensor Array, Surface Response Modeling Algorithms, and Analysis of Variance (ANOVA), IEEE Sensors Applications Symposium(SAS 2008), Atlanta, Feb 12,2008.

4) Clifford K.Ho, Alex Robinson, David R, Miller and Mary J. Davis, Overview of sensors and Need for Environmental Monitoring, Sensors 5, 28 Feb.2005. 